TITLE:

\title{
Cluster size effect on reactive sputtering by fluorine cluster impact using molecular dynamics simulation
}

\section{$\operatorname{AUTHOR}(\mathrm{S}):$}

Aoki, Takaaki; Matsuo, Jiro; Takaoka, Gikan; Yamada, Isao

\section{CITATION:}

Aoki, Takaaki...[et al]. Cluster size effect on reactive sputtering by fluorine cluster impact using molecular dynamics simulation. Nuclear Instruments and Methods in Physics Research Section B: Beam Interactions with Materials and Atoms 2001, 180(1-4): 164-170

\section{ISSUE DATE:}

2001-06

URL:

http://hdl.handle.net/2433/8946

\section{RIGHT:}

この論文は出版社版でありません。引用の際には出版社版をご確認ご 利用ください。; This is not the published version. Please cite only the published version. 
Cluster size effect on reactive sputtering by fluorine cluster impact using molecular dynamics simulation.

T. Aoki, J. Matsuo and I. Yamada ${ }^{*}$

Ion Beam Engineering Experimental Laboratory, Kyoto University, Sakyo, Kyoto, 606-8501, Japan

*) Present Address: Laboratory of Advanced Science and Technology for Industry, Himeji Institute of Technology, CAST, Ako, Hyogo, 678-1205, Japan

e-mail of corresponding author: t-aoki@kuee.kyoto-u.ac.jp

\begin{abstract}
The mechanism of high-yield sputtering induced by reactive cluster impact was investigated using molecular dynamics simulations. Various sizes of fluorine clusters were radiated on clean silicon surface. At an incident energy of $1 \mathrm{eV} /$ atom, $\mathrm{F}$ atom and $\mathrm{F}_{2}$ molecule are only adsorbed on the surface and sputtering of $\mathrm{Si}$ atom does not occur. However, fluorine cluster, which consists of more than several tens molecules causes sputtering. In this case, most of $\mathrm{Si}$ atoms are sputtered as fluorinated material such as $\mathrm{SiF}_{\mathrm{x}}$. This effect is due to the fact that cluster impact induces high-density particle and energy deposition, which enhances both formation of precursors and desorption of etching products. The deposition of atoms and energy becomes denser as the incident cluster size increases, so that larger clusters have shown higher sputtering yield.
\end{abstract}

PACS: 79.20.Rf; 82.65.Pa

Keywords: Molecular dynamics; Fluorine; Silicon; Cluster;

Introduction

Fluorine is one of the most important materials for nano-scale fabrication because of its high chemical reactivity with various materials. As the demand of LSI increases and its scale shrinks, higher etching rate, selectivity and precision are required for etching processes. For the new surface modification techniques, the impact process of clusters on solid surfaces is of great interest [1,2]. Cluster is an aggregated material, which consists of several tens to thousands atoms. When a cluster is accelerated and impacts on a solid target, high-density atomic collision occurs and the kinetic energy of the cluster is deposited on very shallow area of the target. This collisional process is different from that of monomer ions, and is expected to develop into a new surface modification technology. As an example, in the field of sputtering, it has been demonstrated that, when a $\left(\mathrm{SF}_{6}\right)_{2000}$ cluster is irradiated on $\mathrm{Si}$ and $\mathrm{W}$ substrate, the sputtering yield is 10 times higher than that of $\mathrm{Ar}_{2000}$ cluster, which in turn is 10 times higher than monomer ions with the same total acceleration energy of $20 \mathrm{keV} \mathrm{[3].}$

The mechanism of sputtering by reactive cluster ion is not yet completely understood. Molecular dynamics (MD) simulation is one method used to analyze the impact process of ion and solid surface with high space and time resolution. In previous MD work [4], we have shown that a fluorine cluster which consists of 30 fluorine molecules $\left(\left(\mathrm{F}_{2}\right)_{30}\right)$ causes high sputtering yield of the silicon substrate even at low incident energy of $1 \mathrm{eV} /$ atom, whereas a fluorine atom on its' own does not cause sputtering. It is expected that, as cluster size increases and incident particles are radiated with higher density, the surface reaction becomes more enhanced but damage on the surface also increases. Therefore, suitable incident energy and cluster size should be 
considered in order to obtain maximum sputtering yield with least damage. In this paper, we have performed MD simulation of fluorine clusters of various sizes impacting on a silicon surface. The sputtering yield and the sputtered species were investigated in relation to cluster size. The cluster size effect on surface reaction by reactive cluster impact will be discussed.

Simulation model

In this work, molecular dynamics (MD) simulations of fluorine monomer, dimer and cluster impacting on a $\mathrm{Si}(001)$ were performed. In order to describe the interactions between Si-Si, Si-F and F-F, the Stillinger and Weber (SW) type potential model was applied. The SW type potentials were developed by Stillinger et al. [5-7] and modified by Weaklime et al. [8]. Various types of fluorine cluster, with size ranging from 60 to 10000 and incident energy of 1,10 and $100 \mathrm{eV} /$ atom, were prepared. Before the cluster impact, each cluster is annealed in the simulation at $10 \mathrm{~K}$ using the Langevine dynamics method [9]. After annealing, the fluorine cluster has a spherical and amorphous structure, which avoids artifacts due to the structure of the cluster.

The $\mathrm{Si}(001)$ target substrate is simulated as follows: The target consists of maximum 260,000 atoms and its maximum dimension is $180 \AA \times 180 \AA \times 180 \AA$, which is large enough to receive incident energy of this magnitude. The surface $\mathrm{Si}$ atoms construct a stable $2 \times 1$ structure [10]. A periodic boundary condition is applied to the horizontal plane. The Si atoms in the lower four layers are fixed to keep the bulk status and the bottom region of the substrate (up to $1 / 4$ the thickness) is simulated by the Langevine dynamics method to keep the substrate temperature at $300 \mathrm{~K}$.

In order to obtain enough statistics, the simulations of fluorine atom, molecule and small cluster such as $\mathrm{F}_{60}$ were performed 100 times with different impact points. Each impact process is calculated for 8ps, a time interval that is long enough to finish the impact process and investigate the adsorption and desorption of molecules. Through MD simulations of fluorine cluster impact, the sputtering yield of Si and the distribution of sputtered molecules could be examined.

\section{Results and discussion}

Fig. 1 shows the snapshots by MD simulations of $F_{60}, F_{600}$ and $F_{6000}$ clusters impacting on a clean $\mathrm{Si}(001)$ surface. White and black circles represent $\mathrm{Si}$ and $\mathrm{F}$ atoms, respectively. In Fig. 1, each $\mathrm{F}$ atom of the monomer or cluster is accelerated with $1 \mathrm{eV} /$ atom, so the total incident energy increases with increasing cluster size. When the incident energy is as low as $1 \mathrm{eV} /$ atom, $F_{1}$ does not penetrate but is adsorbed on the surface. On the other hand, at the impact of cluster such as $F_{600}$ and $F_{6000}$, the first-impacting fluorine atom, which collides with surface $\mathrm{Si}$ atoms, is accelerated by following $\mathrm{F}$ atoms and penetrates the surface. Consequently, the cluster penetrates the surface and concave damage is formed on the surface. After the impact, most of incident $\mathrm{F}$ atoms evaporate and leave the surface, but some $\mathrm{F}$ atoms remain on the surface forming silicon-fluoride surface with high-density. This high-density fluorination by only one ion impact is a characteristic phenomenon of cluster ion impact.

Fig. 2 shows the snapshots of $F_{60}, F_{600}$ cluster impacts at the same total acceleration energy as $\mathrm{F}_{6000}$ with $1 \mathrm{eV} /$ atom shown in Fig. 1. Fig. 2 also indicates that the impact process of the cluster varies with cluster size. When the cluster size is as small as 60 and each $\mathrm{F}$ atom is accelerated with high energy of $100 \mathrm{eV} /$ atom, the atoms in the 
cluster can penetrate without the acceleration effect created by the following $\mathrm{F}$ atoms, which occurs on large and low-energy cluster impact. Therefore, the whole F atom is implanted into the substrate and multiple collisions are induced in the substrate. Due to the multiple collisions, incident $\mathrm{F}$ atoms spread isotropically, which causes crater-like damage. Crater formation is one of the characteristics of the impact of cluster ions, which was observed in various species of clusters and substrates [11-13]. As can be seen in Fig. 2 , the impact process of $\mathrm{F}_{600}$ with $10 \mathrm{eV} /$ atom resembles that of $\mathrm{F}_{60}$ rather than that of $\mathrm{F}_{6000}$. This result indicates that there is a threshold energy-per-atom, between $1 \mathrm{eV} /$ atom to $10 \mathrm{eV} /$ atom, with which the cluster atoms penetrate the surface and cause multiple collisions to form crater-like damage on the substrate.

Fig. 3 shows the cluster-size dependence of the sputtering yield by $\mathrm{F}$ clusters with 1,10 and $100 \mathrm{eV} /$ atom. At the impact of $F_{1}$ and $F_{2}$, sputtering of Si atoms does not occur at incident energy less than $10 \mathrm{eV} /$ atom, but does occur at $100 \mathrm{eV} / \mathrm{atom}$. In this MD work, different clean surfaces were prepared for each impact, so that it can be considered that the sputtering caused by $\mathrm{F}_{1}$ and $\mathrm{F}_{2}$ requires enough incident energy to knock-on the $\mathrm{Si}$ atoms by elastic collisions. On the other hand, cluster impact causes sputtering even at $\mathrm{F}_{60}$ with $1 \mathrm{eV} /$ atom. As mentioned previously, large cluster irradiation provides large amounts of $\mathrm{F}$ atoms in a narrow region in one impact, so that the formation and desorption of silicon fluoride are considered to be enhanced. As shown in Fig. 3, the sputtering yield increases proportional to $N^{4 / 3}$, where $N$ is the cluster size. If the irradiated $\mathrm{F}$ atoms in the cluster have no interaction with each other, the sputtering yield is simply proportional to $N$. Therefore, this results means that the sputtering yield by cluster impact is enhanced by the factor of $N^{1 / 3}$, which corresponds to the incident density of atom and energy.

Figs. 4(a)-(d) shows the distribution of sputtered particles at the impact of fluorine atom, molecule and clusters of various sizes and incident energy. As can be seen in Fig. 4(a), at the impact of $F_{1}$ and $F_{2}$ with $100 \mathrm{eV} /$ atom, almost all the Si atoms are sputtered as Si monomer, which supports the assumption that sputtering is caused by elastic collisional process at these impacts. On the other hand, the impacts of most of $\mathrm{F}$ clusters, (except for $\mathrm{F}_{60}$ with $100 \mathrm{eV} /$ atom), generate silicon-fluoride species, such as $\mathrm{SiF}$, $\mathrm{SiF}_{2}$ and $\mathrm{SiF}_{3}$, as the major sputtered product, and $\mathrm{Si}$ cluster, $\mathrm{Si}_{\mathrm{x}}$, is a major product for the impact of $\mathrm{F}_{60}$ with $100 \mathrm{eV} /$ atom.

As shown in Fig. 3, at the total incident energy of $6 \mathrm{keV}$, all clusters studied, $\mathrm{F}_{60}$, $\mathrm{F}_{600}$ and $\mathrm{F}_{6000}$, show similar sputtering yield, but the distribution of sputtered particles is different. It is assumed that the mechanism of sputtering differs according to cluster size. As mentioned previously, $\mathrm{F}_{60}$ with $100 \mathrm{eV}$ /atom causes crater-like damage due to the multiple collisions effect. In this crater formation process, a number of atoms on the rim of the crater leave the surface as a cluster, because these atoms have similar kinetic energy and momentum in lateral direction to the surface and the kinetic energy of the cluster is deposited in a shallow region of the surface with high-density [14, 15]. Therefore, a larger number of substrate atoms are sputtered by cluster impact compared to monomer ions. This 'lateral sputtering' occurs not only with F cluster impact but also with Ne cluster impact, which causes only physical collisional process [4]. It can be concluded that the sputtering by $\mathrm{F}_{60}$ with $100 \mathrm{eV} /$ atom is mainly dominated by the physical collision effect, not by chemical effect.

Contrary to $\mathrm{F}_{60}$ impact at $100 \mathrm{eV} /$ atom, the impact of the large cluster, $\mathrm{F}_{6000}$ with $1 \mathrm{eV} /$ atom, shows $\mathrm{SiF}_{3}$ molecules ejected as major sputtered product. When the incident energy is as low as $1 \mathrm{eV} /$ atom, most $\mathrm{F}$ atoms do not penetrate the substrate and cluster-like 
damage is not formed; this impact process is different from that by $\mathrm{F}_{60}$ with $100 \mathrm{eV}$ /atom. However, a large number of $\mathrm{F}$ atoms with high density are provided in the narrow surface region, which contributes to the formation of more volatile molecules with more $\mathrm{F}$ atoms. Considering that the inert $\mathrm{Ne}_{6000}$ cluster with $1 \mathrm{eV} /$ atom does not cause sputtering and only breaks-up on the surface, the sputtering by $F_{6000}$ with $1 \mathrm{eV} /$ atom is due to the chemical reactivity of fluorine and to the high-density particle irradiation effect of the cluster. At the impact of $\mathrm{F}_{600}$ with $10 \mathrm{eV} /$ atom, the ratio of $\mathrm{SiF}$ and $\mathrm{SiF}_{2}$ to total sputtered products increases compared to $\mathrm{F}_{6000}$ with $1 \mathrm{eV} /$ atom. This is because $\mathrm{F}_{600}$ provides less $\mathrm{F}$ atoms compared to $\mathrm{F}_{6000}$, but dense kinetic energy deposition enables Si atoms with less $\mathrm{F}$ atoms, such as $\mathrm{SiF}$, to be sputtered easily. From the results at total incident energy of $6 \mathrm{keV}$, it is expected that high sputtering yield with shallow damage can be achieved using large reactive cluster.

As shown in Fig. 4(c), when the total incident energy is $600 \mathrm{eV}$, the impact of $\mathrm{F}_{600}$ with $1 \mathrm{eV}$ shows $\mathrm{SiF}_{3}$ as the major sputtered product, whereas for $\mathrm{F}_{60}$ with $10 \mathrm{eV}$ /atom $\mathrm{SiF}$ is dominant. This result indicates that the cluster size of 600 gives enough dense irradiation of $\mathrm{F}$ atoms to form $\mathrm{SiF}_{3}$ molecules, whereas this density is apparently not sufficient with cluster size of 60 , and mainly $\mathrm{SiF}$ and $\mathrm{SiF}_{2}$ are formed instead, and these compounds in turn can be sputtered due to the higher-density irradiation energy of $10 \mathrm{eV} /$ atom. For similar reasons the distribution by $\mathrm{F}_{60}$ with $1 \mathrm{eV} /$ atom shown in Fig. 4(d) can be explained. $\mathrm{F}_{60}$ with $1 \mathrm{eV} /$ atom sputters mainly $\mathrm{SiF}_{2}$. This is because that the incident energy of $1 \mathrm{eV} /$ atom is not enough for $\mathrm{SiF}$ to be desorbed but sufficient for $\mathrm{SiF}_{2}$. From these results, the dependency of sputtered products on cluster size and incident energy is summarized as follows: Large clusters with the size 600 or more can generate $\mathrm{SiF}_{3}$ and $\mathrm{SiF}_{4}$, which are easily desorbed at low energy of $1 \mathrm{eV} /$ atom. On the other hand, $\mathrm{F}_{60}$ impacts generates sufficient $\mathrm{F}$ irradiation density and the desorption of these incomplete compounds depends in turn on the radiated energy density; $\mathrm{SiF}_{2}$ can be desorbed with only $1 \mathrm{eV} /$ atom, but $\mathrm{SiF}$ needs a higher energy of $10 \mathrm{eV} /$ atom for its' desorption.

Summary and Conclusions

Molecular dynamics simulations of reactive cluster impacts were performed in order to investigate the cluster size effect on the sputtering process. For the same incident energy-per-atom, the total sputtering yield per cluster impact increases in proportion to the $4 / 3$ power of the cluster size, $N$. This result means that, compared to the sputtering yield of independent impacts of same number of $F$ atoms as the cluster, the sputtering yield by the cluster is enhanced by the factor $N^{1 / 3}$, which corresponds to the incident density of energy and particles given by the cluster impact. At the impact of clusters with the same total acceleration energies of $6 \mathrm{keV}$, the total sputtering yield shows similarities between various cluster sizes. However, the penetration depth and the distribution of sputtered products are different for different cluster sizes. A small cluster with high incident energy-per-atom, such as $\mathrm{F}_{60}$ with $100 \mathrm{eV} /$ atom, penetrates the surface deeply and causes crater-like damage on the surface. In this collisional process, the substrate $\mathrm{Si}$ atoms are sputtered out mainly as Si cluster, not in Si-F compounds. This sputtering mechanism by such cluster is similar to that by inert Ne cluster with same size and incident energy, and therefore, is mainly dominated by physical collisional process. On the other hand, at the impact of large cluster with low energy like $\mathrm{F}_{6000}$ with $1 \mathrm{eV} / \mathrm{atom}$, the cluster does not penetrate the surface but a large number of silicon-fluorides are generated because of the high-density incidence of $\mathrm{F}$ atoms, so that most $\mathrm{Si}$ atoms are desorbed as 
$\mathrm{SiF}_{3}$ compounds. From these results, it is expected that high-yield and low damage etching can be achieved by impact of large reactive cluster consisting of several thousands atoms.

\section{References}

[1] I. Yamada, J. Matsuo, N. Toyoda, T. Aoki, E. Jones and Z. Insepov, Mater. Sci. and Eng., A253 (1998) 249

[2] I. Yamada, J. Matsuo, Z. Insepov, T. Aoki, T. Seki and N. Toyoda, Nucl. Instr. and Meth. B, 164-165 (2000) 949

[3] N. Toyoda, H. Kitani, N. Hagiwara, J. Matsuo and I. Yamada, Mater. Chem. and Phys., 54 (1998) 106

[4] T. Aoki, J. Matsuo and I. Yamada, Nucl. Instr. and Meth. B, 164-165 (2000) 546

[5] F. H. Stillinger and T. A. Weber, J. Chem. Phys., 88 (1988) 5123

[6] F. H. Stillinger and T. A. Weber, Phys. Rev. Lett., 62 (1989) 2144

[7] T. A. Weber and F. H. Stillinger, J. Chem. Phys., 92 (1990) 6239

[8] P. C. Weakliem, C. J. Wu and E. A. Carter, Phys. Rev. Lett., 69 (1992) 200

[9] W. F. van Gunstern and H. J. C. Berendsen, Molec. Phys., 45 (1982) 637

[10] H. Feil and H. J. W. Zandvliet, M. -H. Tsai, J. D. Dow and I. S. T. Tsong, Phys. Rev. Lett., 69 (1992) 3076

[11] V. I. Shulga and P. Sigmund, Nucl. Instr. and Meth. B, 62 (1991) 23

[12] C. L. Cleveland and U. Landman, Science, 257 (1992) 355

[13] J. Nordiek, M. Moseler and H. Haberland, Rad. Eff. and Def. in Solids, 142 (1997) 27

[14] T. Aoki, J. Matsuo, Z. Insepov and I. Yamada, Nucl. Instr. and Meth. B, 121 (1997) 49

[15] N. Toyoda, H. Kitani, N. Hagiwara, T. Aoki, J. Matsuo and I. Yamada, Mater. Chem. and Phys., 54 (1998) 262 


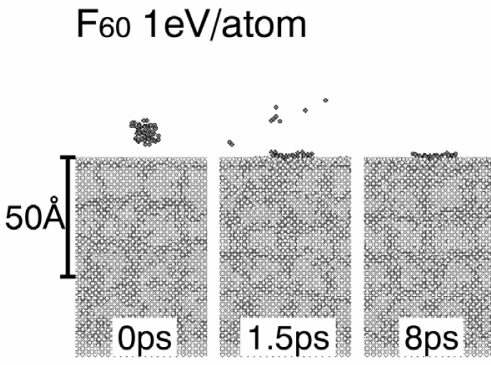

F600 1eV/atom

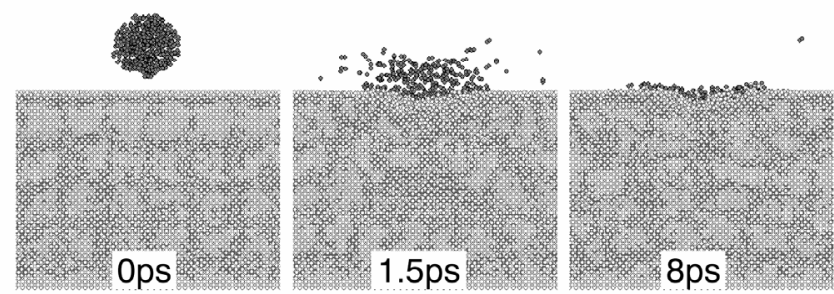

\section{F6000 1eV/atom}
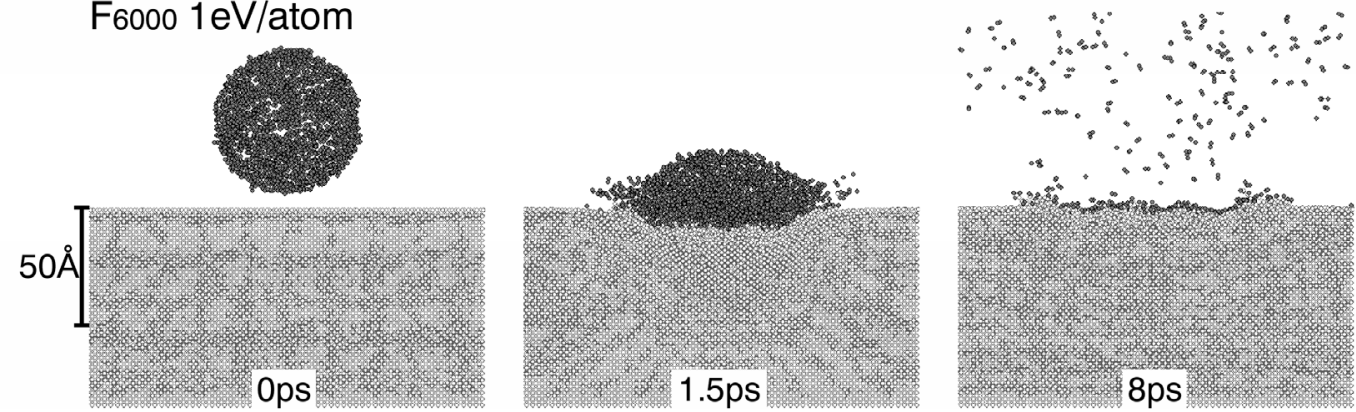

Fig. 1: Snapshots of MD simulations of $F_{60}, F_{600}$ and $F_{6000}$ with $1 \mathrm{eV} /$ atom impacting on clean surface on clean $\mathrm{Si}(001)$ surface. Black and white circles represent $\mathrm{F}$ and $\mathrm{Si}$ atoms, respectively. 


\section{F60 100eV/atom}

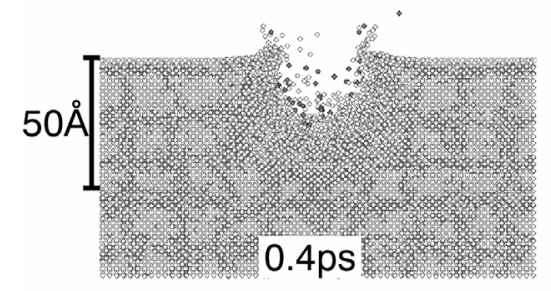

F600 10eV/atom
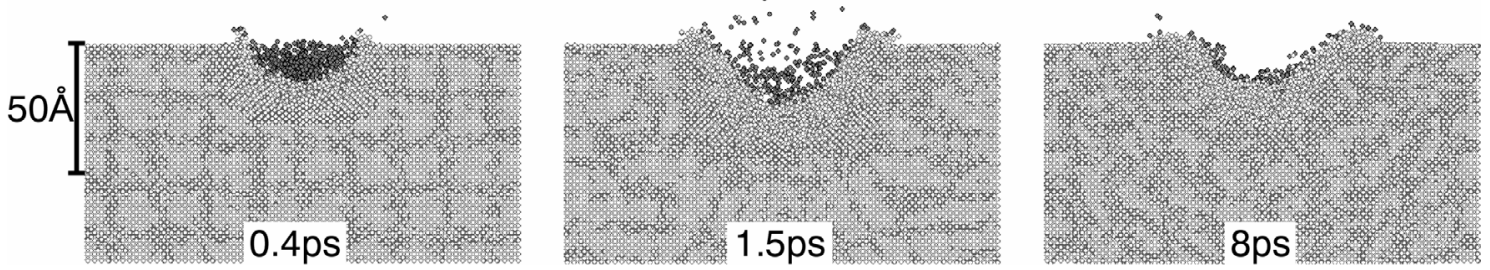

Fig. 2: Snapshots of MD simulations of $F_{60}$ with $100 \mathrm{eV} /$ atom and $F_{600}$ with $10 \mathrm{eV} /$ atom. These clusters have similar total incident energy to $\mathrm{F}_{6000}$ with $1 \mathrm{eV} /$ atom shown in Fig. 1. 


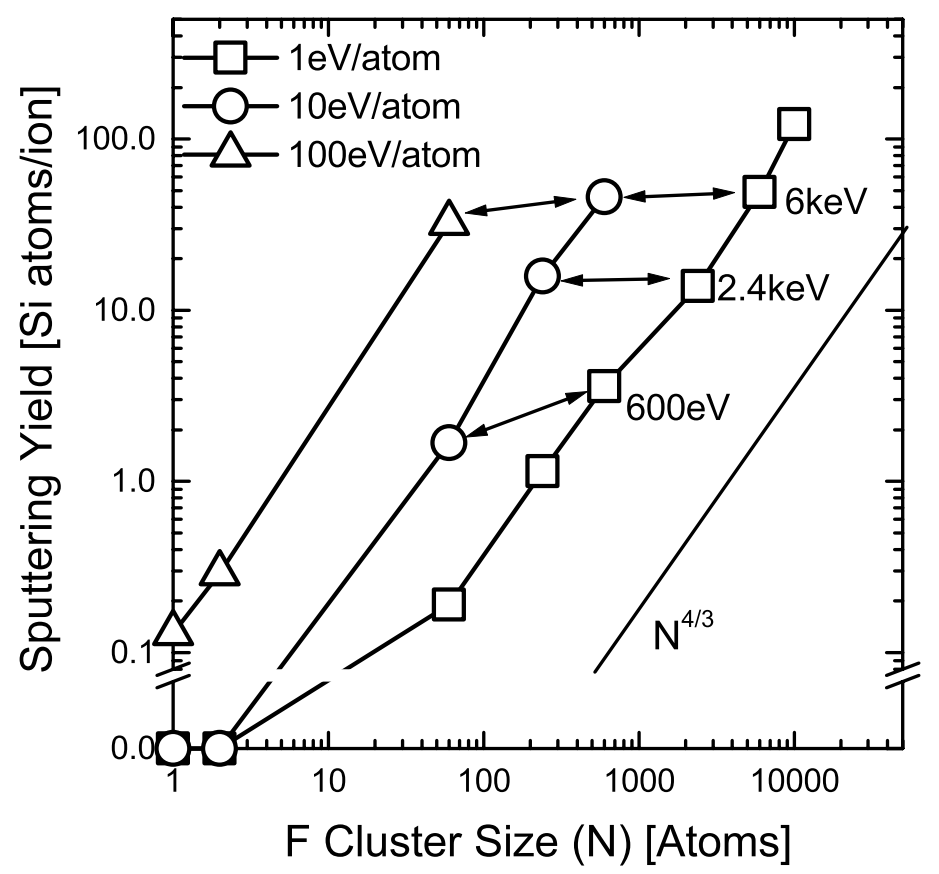

Fig. 3: Cluster-size dependence of total sputtering yield of Si atoms. 

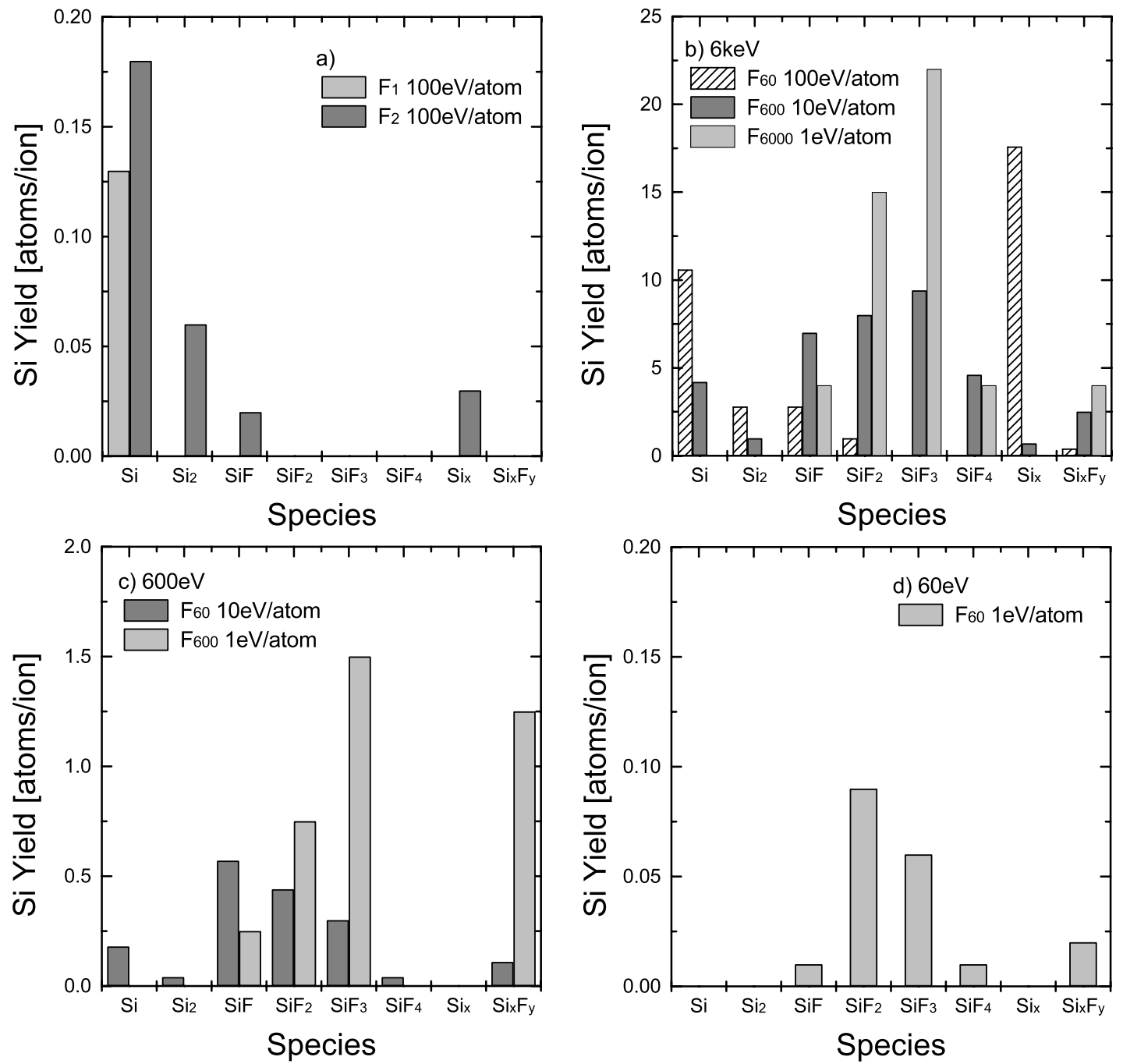

Fig. 4: Distributions of sputtered products induced by $F_{1}$ and $F_{2}$ with $100 \mathrm{eV} /$ atom (a) and F clusters with total incident energy of $6 \mathrm{keV}(\mathrm{b}), 600 \mathrm{eV}$ (c) and $60 \mathrm{eV}$ (d). 